\title{
Requirement Analysis of Product Line Based Semantic Web Services
}

\author{
Nakul C. Das, Shamim Ripon, Senior Member, IACSIT, Orin Hossain, and Mohammad Salah Uddin
}

\begin{abstract}
These Web services technology provides a platform on which we can develop distributed application by using existing service oriented application. Software product line on the other hand provides a systematic mechanism to manage the reusable components of the product family within the product line. In this work we followed an approach to combine these two approaches to achieve the benefits of both. The common and variants features of software product line can be modeled by using a feature diagram. We first perform a commonality and variability analysis of the requirements of the product line. From such analysis we develop a dependency graph consisting of the features and constraints among them. A walk through the graph can solve the constraints. We draw goal models of various functional features of the product line to get a clear view whether a certain goal can be achieved by the product line model. In order to check the consistency of the feature model we encode the feature by using OWL-DL. Such encoding facilitates to check whether any valid product can be composed from the product family model. We perform our analysis and experiment by using a Travel Agent Web Service.
\end{abstract}

Index Terms-Semantic web, web services, OWL-DL, feature model, product line, web service composition.

\section{INTRODUCTION}

Semantic Web Services is an emerging technological concept that combines technologies from Web Services, the Semantic Web, and automatic reasoning. Semantic Web Services emphasizes enabling dynamic, execution-time discovery, composition, and invocation of Web Services [1] allowing automated ad-hoc interaction between web-based applications [2]. It builds on the existing Web Services technology that provides software developers a framework for accurate documentation of services to allow the development-time discovery, composition, and statically coded invocation of services in the Web. The aim of Semantic Web Services technology is to automate these tasks that currently require human software developers.

The predicted advantages of the Semantic Web Services can be achieved from up-to-date, comprehensive, and effective utilization of Web Services. As the decision to use a particular service can be made at the execution time instead of development time of the service requestor software, it is possible to use services that are not available, or even existing, at development time but are only discovered at execution time. This will dramatically improve the usability of Web Services technology in volatile environments, such as in mobile and ubiquitous computing, where the landscape of available services can change rapidly, for example, when a

Manuscript received September 9, 2013; revised December 31, 2013.

The authors are with the Department of Computer Science and Engineering, East West University, Bangladesh (e-mail: dshr@ewubd.edu). device moves from one space to another. Such benefits can also be made available to non-mobile open environments, such as the Web where the available services evolve over the time and many different kinds of services may be available to satisfy the particular goals.

Semantic Web [3] can be used to represent the description of services in a precisely machine understandable form which could form the basis of automatic and execution time reasoning about the type, structure and properties of services. Other reasoning methods, such as logical reasoning can be combined with semantic web not only to describe services in accurate manner but also to discover promising services and composing them into complex workflows, and to determine the correct way to invoke them

The Semantic Web and Web Services are two complementary initiatives, building on the notational ground provided by XML. Semantic Web is declarative representation approach to describe domain information in machine processable way, while Web Services provide a mechanism to invoke services across the Web in a way that resembles remote procedure calls.

The central parts of Web Services technology are descriptions of the interfaces of services specified in Web Services Description Language (WSDL) [4] to make it possible to invoke them over the Internet. There are a number of additional specifications dealing with many other aspects of Web Service use, namely, orchestration, choreography, addressing, security, resource states, and so on.

Software product line is a set of software intensive systems sharing a common, managed set of features that satisfy the specific needs of a particular market segment or missions and that are developed from a common set of core assets in a prescribed way [5]. The main idea of software product line is to explicitly identify all the requirements that are common to all members of the family as well as those that vary among products in the family. A particular product is then derived by selecting the required variants and configuring them according to the product requirements.

Software product line and web service composition share some similarities among them. A web service provider has a set of specified product features that have to be fulfilled by all the possible service composition provided to users. On the contrary, some services are not required by all the service composition but included in a few composition based on user requirements.

The objective of this work is to apply software product line techniques to manage the service composition and reuse of services across the composed services. The reuse mechanism of software product line can be successfully applied to web services. In this paper we present a systematic mechanism that allows us to analyze the service composition in the light of software product line. We perform a case study of Travel 
Agent web service.

In order to understand the available features that can be reused during various web services composition, we present a commonality and variability analysis (CVA) of the Travel agent web service. Such analysis portrays clearly the common and variant features of the said web service and depicts the dependencies and constraints of the services. A goal model checks whether the user objectives or goals can be accomplished from the composed services. We draw goal model for the common functionalities of travel agent web service. Besides analyzing the service composition, we also verify the feature relations of the web services. We model the features using semantic web technique OWL-DL [6] and verify the feature composition using Protégé [7] and RacerPro [8].

In the rest of the paper, Section II gives a brief description of Travel Agent Web Service. The common and variants features of the web service are illustrated by using feature diagram. We then describe our analysis mechanism of our case study in Section III. The semantic web based verification of feature composition is illustrated in Section IV Finally we conclude our paper and outline our future plan in Section V.

\section{Travel Agent Web Services OVERVIEW}

A Travel Agent Web Service is a system for booking tickets for train, bus, air, cruise etc. as well as for booking accommodation system. The basic operational scenarios are similar in all the Travel AgentWeb Service systems. However, there are many variants on the basic theme, e.g., holiday packages can be provided by some Travel Agent Web services while some others only provide basic ticket services

\section{A. Travel Agent Domain Model Using FODA}

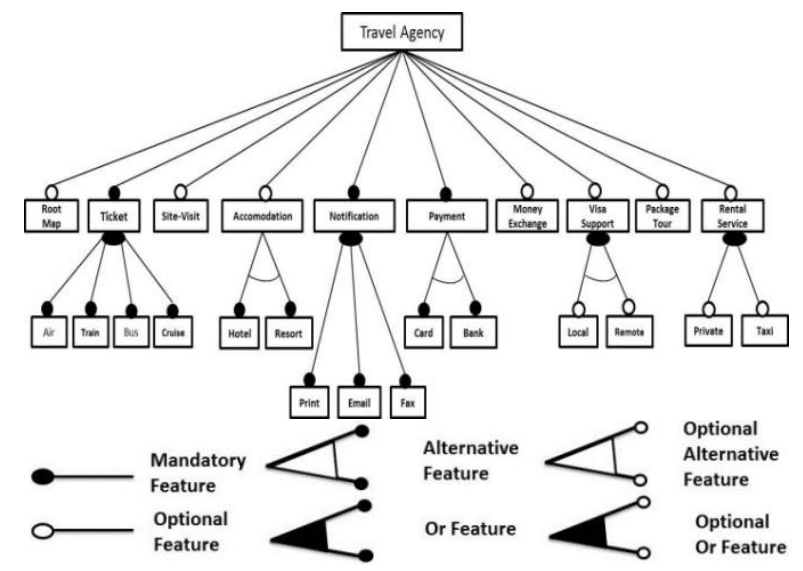

Fig. 1. Travel agent web service feature diagram.

Modeling variants is an important process during designing software product line. The feature oriented domain analysis (FODA) [9] focuses on identifying features that characterize a domain. Features are user visible aspects or characteristics of a system and are organized into And/Or graph in order to identify the commonalities and variants of the application domain. Feature modeling is an integral part of the FODA method and the Feature Oriented Domain Reuse Method (FORM) [10]. The commonalities and variants within features are exploited to create a set of models that is used to implement any member product of that family.

In feature tree, features are represented in graphical form as trees. The internal nodes of a tree represent the variants and their leaves represent values of corresponding variants. Graphical symbols are used to indicate the categories of features. The feature diagram depicts the classification of mandatory features and variant features as well as their dependencies. Mandatory features are those which are present in all products in the respective domain. Variant features appear only some members of the domain which differentiate one product from others.

A partial feature tree of Travel Agent Web Service System is shown in Fig. 1. Extensions of feature diagram described in [11] have been used here. Variant features are also classified as Optional, Alternative and $\mathrm{Or}$ features. An example of optional feature is 'Accommodation System'. An alternative feature describes one of many features. An example of alternative feature is 'Payment System' which can be either Bank Transfer System or Card System. An or-feature describes any of manyfeatures.For example a 'Ticket Booking' can be made by multiple services or multiple times or by both. Variants may depend on other variants. Two types of dependencies are illustrated in this paper.

\section{ANALYSIS OF SERVICE COMPOSITION}

Our approach contains two workflows, one for the web service product developer and another for the user. This approach provides a two-stage process. From the point of view of product line development, the development process is divided into the domain engineering phase and the application engineering phase, whereas from the point of view of web service design, the process is divided into the preparation stage (before implementation) and the customization stage (after implementation). An overview of our approach is illustrated in Fig. 2.

At the top half of Fig. 2, i.e., the domain engineering phase, the developer performs a commonality and variability analysis (CVA) of the system requirements. The results of the CVA include a formalized specification of all the common and variable functional properties. Each variable has some associated parameters to configure, called parameters of variation. Some of these parameters may have dependencies or tradeoffs with other parameters of variation. We model this dependency of variations in a variability dependency graph.

Each commonality and parameter of variation is also associated with a set of service compositions that satisfy them. We call this relation the mapping-relation. The results of the CVA are captured in a product-line decision model. Given the system requirements, the developer can identify the component web services that are relevant to the domain and the system.

For the application engineering phase, the services retrieved from the service broker are composed according to the common functional requirements. The composition set is then pruned to a smaller set in which each composition satisfies all the commonalities. This set serves as the composition search space for the variability, and we call it the commonality composition set.

After implementation of the web services, the user can 
access a default web service with basic functionalities. The user can then customize the functional properties by setting all the parameters of variation in the decision model. By interacting with the decision model, the input requirements from the user are always kept consistent through solving the constraints in the variability dependency graph. The consistent specification of the variabilities can be fed into a query algorithm to search valid compositions in the composition search space.

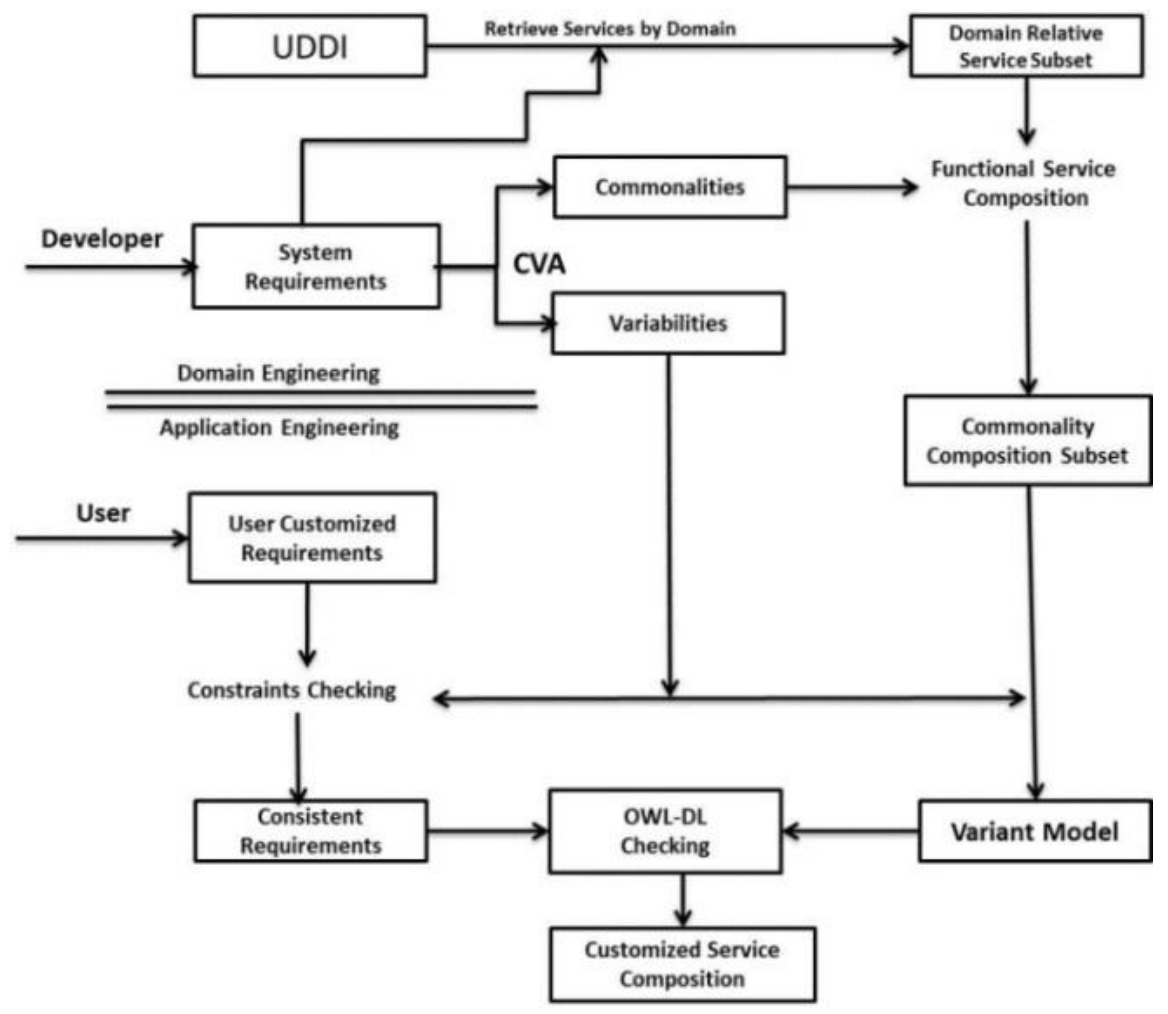

Fig. 2. Overview of analysis approach.

Travel Agent Web Service consists of several different services. The web service can also invoke other third party services. Fig. 3 shows a sample structure for Travel Agent service composition. The services are connected by the dashed lines represent the optional services in a valid composition.

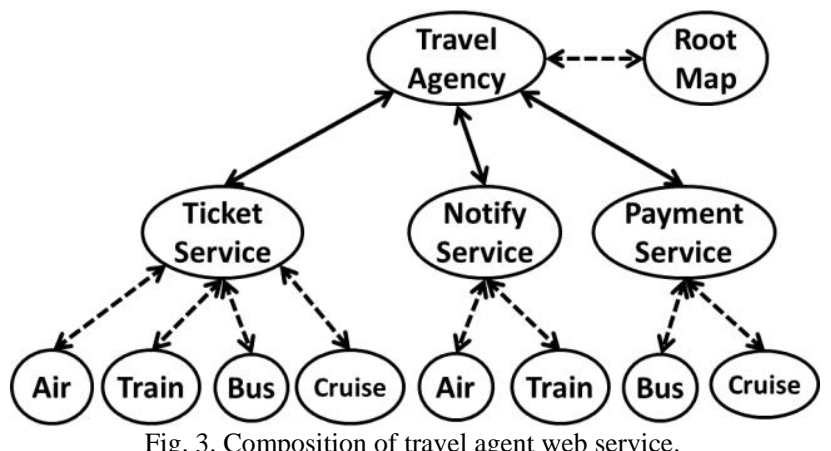

\section{A. Commonality and Variability Analysis (CVA)}

The commonalities and variabilitiesof the Travel Agent service compositions are listed according to their label, type (Functional requirements as "FR"), their description and their service mappings and parameter(s) of variation and any dependencies among these parameters (Fig. 4).

\section{B. Dependency Graph}

We model the dependencies of the parameters in the CVA in a dependency graph (Fig. 5). In the dependency graph, each node represents variability and contains the information of all the parameters related to this variability. The edges between the nodes represent and contain the constraints between the two variabilities. The graph may not be fully connected. A graph-walk algorithm can be used to traverse the sub-graphs in a random order or a userpreferred order to solve all the constraints.

Example Commonality:

- $\mathrm{C} 1$ :

$\circ$ FR

- There must be a Travel Agent Service.

- Travel Agent Service $=$ any service in Travel Agent Service set $\{\mathrm{TA} 1, \mathrm{TA} 2, \mathrm{TA} 3 \ldots\}$

- $\mathrm{C} 2$

○ FR

- There must be one or more Ticket Service.

- Ticket Service $=$ any service in Air Ticket Service set $\{$ ATS1, ATS2, ... $\}$ or Train Ticket Service set $\{$ TTS1,TTS2, ... $\}$ or Bus Ticket Service set $\{$ BTS1,BTS2, ... $\}$ or Cruise Ticket Service set $\{\mathrm{CTS} 1, \mathrm{CTS} 2, \ldots\}$.

Example Variability:

- V1:

$\circ$ FR

- Existence of Air Ticket service

$\circ\{$ True, False

$\circ$ If V1 is False, (V2 or V3 or V4) is True

- V2:

o FR

- Existence of Train Ticket service

$\circ$ \{True, False

o If V2 is False, (V1 or V3 or V4) is True 


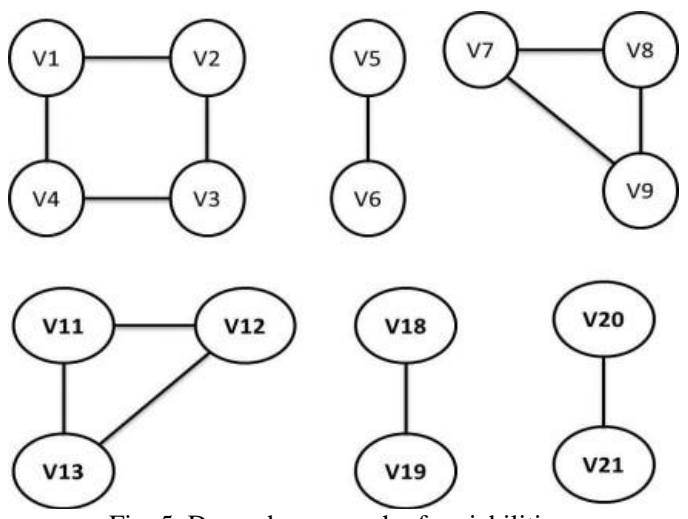

Fig. 5. Dependency graph of variabilities.

\section{Goal Model}

In order to generate all the service compositions that satisfy the commonalities, we need to represent the common functionalities, in the goal model. Fig. 6 shows the goal model for the common functionalities. Note that we have preserved the possibility of using third-party services to implement the functionality ThirdPartyservice (OtherData) in the goal model. The functionalcomposition process finds appropriate componentservices to implement these abstract methods in the goal model in accordance withthe service mapping table. This service mapping table is constructedtogether with the goal model by looking up the CVA results. For example, There is a mapping: ThirdPartyservice = any element from $\{\mathrm{N} / \mathrm{A}, \mathrm{GPS}-\mathrm{MAP}, \mathrm{TP} 1, \mathrm{TP} 2, \ldots\}$.

\section{SEMANTIC Web BASED ANALYSIS}

By using OWL-DL we model six types of relations, namely mandatory, optional, alternative, or, optional alternative and optionalor. Two additional constraints: requires and excludes are also modeled. Modeling feature models using OWL-DL have several advantages, such as facilitating feature model storing, sharing and distributing and assisting cooperative designing.

First, OWL ontology is built for various nodes and edges in the feature model. The ontology is constructed in three steps:

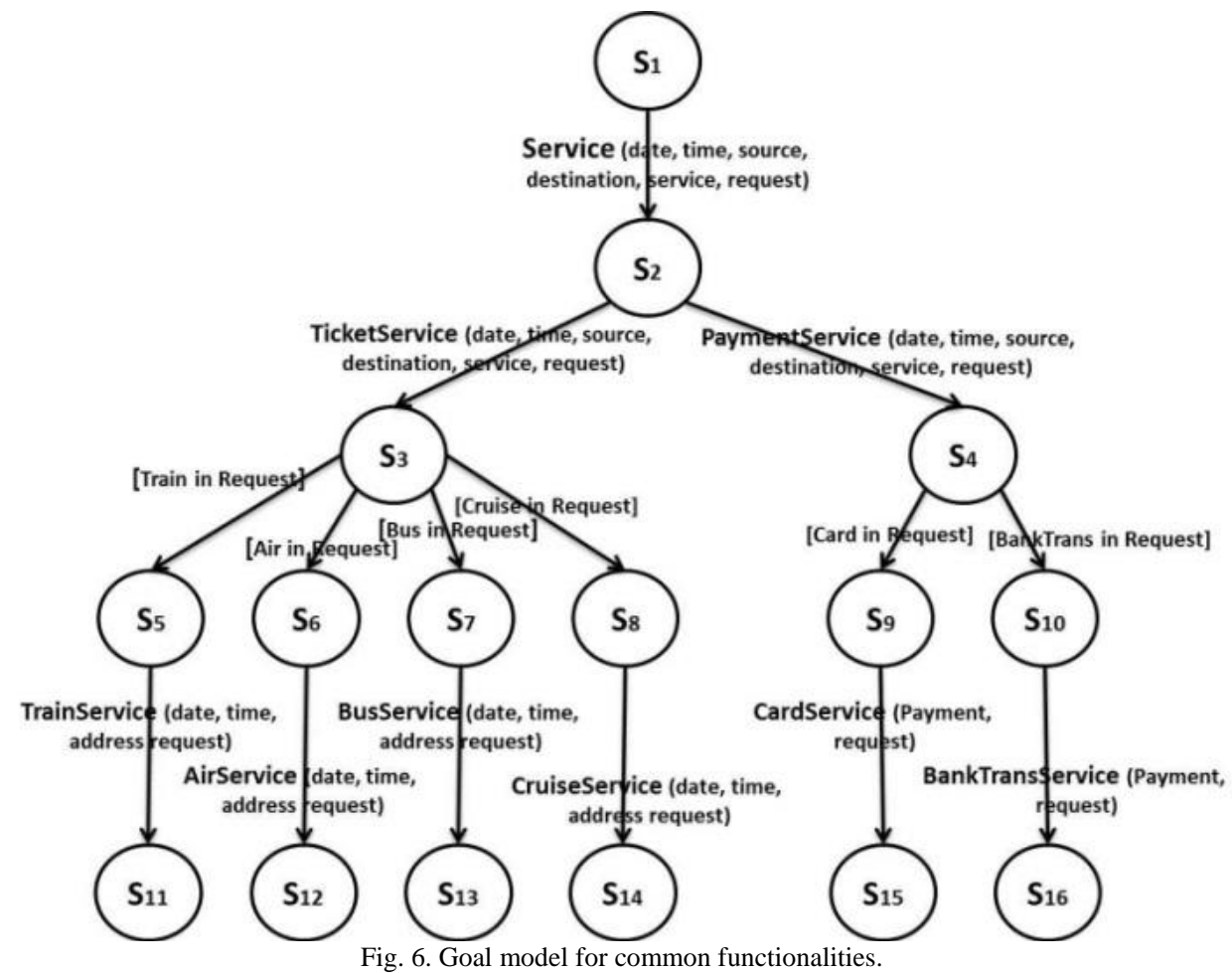

Step1: We identify the nodes (concepts and features) present in a feature diagram. Each node in the diagram is modeled as an OWL class. Moreover, we assert that these classes are mutually disjoint. In OWL, all classes are assumed to overlap unless it is otherwise stated that they are disjoint with each other using a disjoint axiom.

Step 2: For each node, we create a Rule class. This Rule class has two kinds of conditions: firstly, a necessary and sufficient (NS, EquivalentClass) condition, using an existential restriction to bind the Rule node to the corresponding feature node in the diagram; and secondly, a number of (possibly 0) necessary ( $\mathrm{N}$, subclassof) constraints later, serving two purposes: 1) to specify how each of its child features is related to this node, capturing the various relations between features, 2) to specify how this feature node is constrained by other features, in the form of requires, excludes, Optional Alternative and Optional Or.

Step 3: The root concept and features in a feature diagram are interrelated by various feature relations, represented by different edge types in the diagram. In our OWL model, for each of these edges, we create an object-property. We assert that the range of the property is the respective feature class.

The OWL syntax used in this paper is summarized in Table I. For a parent feature $A$ and for each of its child features $B_{1}, B_{2}, \ldots, B_{n}$ the initial modeling produces the following ontology.

$$
A \sqsubseteq \mathrm{T} \quad \text { has } \subseteq \text { ObjectProperty }
$$




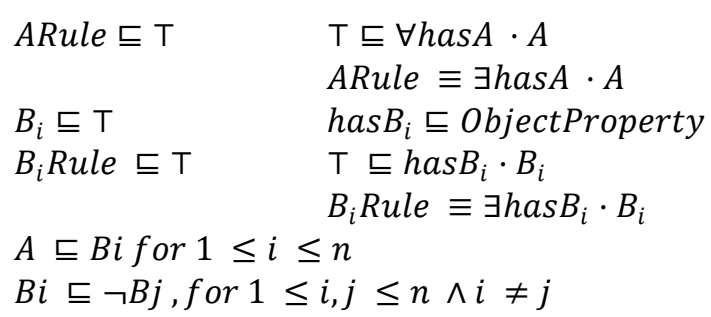

We can now model various types of feature relations by using ontology.

TABLE I: SUMMARY OF OWL-DL SYNTAX

\begin{tabular}{lc}
\hline Notation & Explanation \\
\hline $\mathrm{T}$ & Superclass of all OWL classes \\
$A \subseteq B$ & $A$ is a subclass of $B$ \\
$A \subseteq \neg B$ & $A$ and $B$ are disjoint \\
$A \sqcap B$ & Class intersection \\
$A \sqcup B$ & Class union \\
$A \equiv B$ & Class equivalence \\
$\mathrm{T} \subseteq \forall P \cdot A$ & Range of property is a class $A$ \\
& allValuesFrom/someValuesFrom restriction, giving \\
$\mathrm{T} \subseteq \exists P \cdot A$ & the class that for every instance of this class that has \\
& instances of property $\mathrm{P}$, all/some of the values of the \\
& property are members of the class A
\end{tabular}

\section{Modeling VArious Types of Features}

\section{A. Mandatory}

A mandatory feature is included if its parent feature is included.For each of the mandatory features $B_{1}, B_{2}, \ldots, B_{n}$ of a parent feature $A$, we use one $N$ constraints in ARule to model it. It is a someValuesFrom restriction on has $_{i}$, stating that each instance of the rule class must have some instance of $B_{i}$ class for $h a s B_{i}$. The following ontology fragment shows the modeling of mandatory feature set and parent feature A.

$$
\text { ARule } \sqsubseteq \exists h a s B_{i} \cdot B_{i} \text { for } 1 \leq i \leq n
$$

It can be seen fromFig. 1 that the root nodehas a mandatory child feature Payment Service, which is itself a non-leaf node. We create two new classes (parent and child) for these two non-leaf nodes.

\section{For parent feature:}

TravelAgent $\subseteq \mathrm{T}$
hasTravelAgent $\sqsubseteq$ ObjectProperty
TravelAgentRule $\subseteq \mathrm{T} \subseteq \square$ hasTravelAgent $\cdot$ TravelAgent
TravelAgentRule $\equiv \exists$ hasTravelAgent $\cdot$ TravelAgent

\section{For child feature:}

PaymentService $\sqsubseteq \mathrm{T}$

PaymentServiceRule $ᄃ \mathrm{~T}$

hasPaymentService $\sqsubseteq$ ObjectProperty

$\mathrm{T} \sqsubseteq$ hasPaymentService · PaymentService

PaymentServiceRule $\equiv$

TravelAgent Rule $\sqsubseteq$

$\exists$ hasPaymentService $\cdot$ PaymentService

$\exists$ hasPaymentService $\cdot$ PaymentService

\section{B. Optional}

An optional feature may or may not be included in a diagram, if its parent is included. For each of the optional features $B_{l}$, $B_{2}, \ldots, B_{n}$ of a parent feature $\mathbf{A}$, no additional statements are required to model this relationship. In that case they are denoted by,

$$
\begin{aligned}
& B i \sqsubseteq \mathrm{T} \quad \text { hasBi } \sqsubseteq \text { ObjectProperty } \\
& \text { BiRule } \sqsubseteq \mathrm{T} \quad \text { BiRule } \equiv \exists \text { has Bi } \cdot \mathrm{Bi}
\end{aligned}
$$

Accommodation service is an optional feature ofTravel Agent Web Service and itmay, or may not be included in a configuration of Hall Booking System. Asit is a non-leaf node, we create one new class AccomService for Accommodation Service Reservation Charge and one object-property hasAccomService. The ontology is augmented as follows. Note that no new restriction on TravelAgent is added.

\section{AccomService $\sqsubseteq \mathrm{T}$ \\ hasAccomService $\sqsubseteq$ ObjectProperty AccomServiceRule $\sqsubseteq \mathrm{T}$ AccomServiceRule $\equiv$ $\exists$ GasAccomService · AccomService}

\section{Alternative}

One and only one feature from a set of alternative features can be included, if their parent feature is included in a configuration. Hence, for a set of alternative features $B i$ and $B j$ are two children of parent feature $A$, we use disjunction of someValuesFrom restrictions over hasBi and has $B j$ to ensure that some feature will be included. We use the complement of distributed disjunction of the conjunction of two someValuesFrom restrictions to ensure that only one feature can be included. The 'or' symbol $\sqcup$ represents distributed disjunction.

$$
\begin{aligned}
& \text { ARule } 匚\left(\left(\exists h a s B_{i} \cdot B_{i}\right) U(\exists h a s B j \cdot B j)\right) \\
& \text { for } 1 \leq i \leq n \text { and for } 1 \leq j \leq n \\
& \text { ARule } \neg \neg\left(\left(\exists h a s B_{i} \cdot B_{i}\right) \Gamma(\exists h a s B j \cdot B j)\right) \\
& \text { for } 1 \leq i \leq n \text { and for } 1 \leq j \leq n
\end{aligned}
$$

Fig. 1 shows that features CardService and BankTrnsService are alternative features of Payment. We model this relation as follows.

\section{PaymentService $ᄃ \mathrm{~T}$}

hasPaymentService ᄃ ObjectProperty

PaymentServiceRule $\sqsubseteq \mathrm{T}$

$\mathrm{T} \subseteq \forall$ hasPaymentService $\cdot$ PaymentService

PaymentServiceRule

$$
\begin{aligned}
& \equiv \exists \text { hasPaymentService } \\
& \text { - PaymentService }
\end{aligned}
$$

\section{CardService $\sqsubseteq \mathrm{T}$}

hasCardService $\sqsubseteq$ ObjectProperty

CardServiceRule $ᄃ \mathrm{~T}$

$\mathrm{T} \sqsubseteq$ has CardService · CardService

$$
\text { CardServiceRule }
$$

PaymentServiceRule

$$
\equiv \exists \text { has CardService } \cdot \text { CardService }
$$

드 hasCardService $\cdot$ CardService

\section{BankTrnsService ㄷ T hasBankTrnsService}

$$
\sqsubseteq \text { ObjectProperty }
$$


BankTrnsServiceRule $\sqsubseteq \mathrm{T}$

$\mathrm{T} \sqsubseteq$ hasBankTrnsService · BankTrnsService

BankTrnsServiceRule

$$
\begin{aligned}
& \equiv \exists \text { has BankTrnsService } \\
& \cdot \text { BankTrnsService }
\end{aligned}
$$

PaymentServiceRule

$$
\sqsubseteq \exists \text { has BankTrnsService }
$$$$
\text { - BankTrnsService }
$$

PaymentServiceRule 드

( $(\exists$ hasCardservice $\cdot$ Cardservice $)$

$\sqcup$ ( $\exists$ hasBankTrnsService

- BankTrnsService))

PaymentServiceRule 드

$\neg((\exists$ hasCardservice $\cdot$ Cardservice $)$

$\sqcap$ ( $\exists$ hasBankTrnsService

- BankTrnsService))

The last two restrictions ensure that one and only one feature from the set of alternative features can be included.

D. $\mathrm{Or}$

At least one from a set of available features is included, if the parent feature is included. For a set of $O r$ features $B_{1}$, $B_{2}, \ldots, B_{n}$ of a parent feature $A$, we need to use a disjunction of someValuesFrom restrictions to model this relation.

$$
\text { ARule } \sqsubseteq U(\exists h a s B i \cdot B i), \text { for } 1 \leq i \leq n
$$

It may be noticed that the definition of Or is very similar to that of Alternative, with the omission of the negation of distributed disjunction to allow for multiple or features to be included. In Fig. 1, the feature Reservation Charge has a number of $\mathrm{Or}$ features. We use the following constructs ${ }^{1}$ to model it.

\author{
TicketService 드 $\mathrm{T}$ \\ hasTicketService 다 ObectProperty \\ TicketService Rule $\sqsubseteq \mathrm{T}$ \\ TicketService Rule 드 \\ $\exists$ ヨasTicketService $\cdot$ TicketService \\ AirService $\sqsubseteq \mathrm{\top}$ \\ hasAirService Oㅣ ObectProperty \\ AirServiceRule $\sqsubseteq \mathrm{T}$ \\ $\top \sqsubseteq$ hasAirService $\cdot$ AirService \\ AirServiceRule $\equiv \exists$ hasAirService $\cdot$ AirService \\ TicketService Rule 드 ヨhasAirService · AirService \\ ..... \\ TicketService Rule \\ 드 (( ĐhasAirService $\cdot$ AirService $)$ \\ $\sqcup(\exists$ hasTax $\cdot$ Tax $)$ \\ $\sqcup$ ( hasBasicCharge $\cdot$ BasicCharge) \\ $\sqcup$ (ヨhasDiscount $\cdot$ Discount)
}

\section{E. Optional Or}

One or more optional feature may be included when parent feature is included. There is no Optional Or feature in our

${ }^{1}$ For brevity, definitions of Rule classes are omitted from here. brief case study but a detailed feature model consists of such features. If $A$ is parent feature of child features $B$ and $C$. The OWL-DL representation is defined as follow:

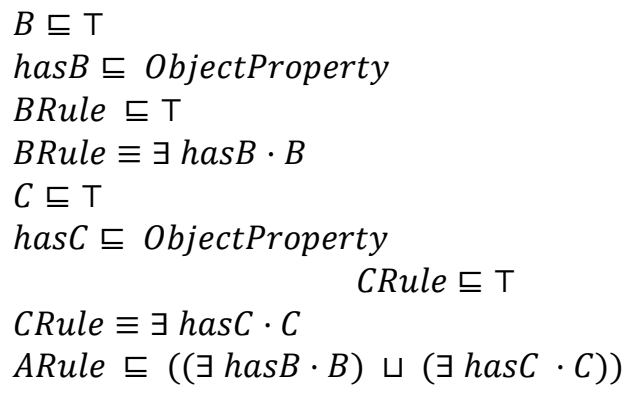

\section{F. Optional Alternative}

One feature from a set of alternative features may or may not be included if parent is included. Consider $A$ is parent feature of child features $B$ and $C$. We can write the OWL-DL representation as follow:

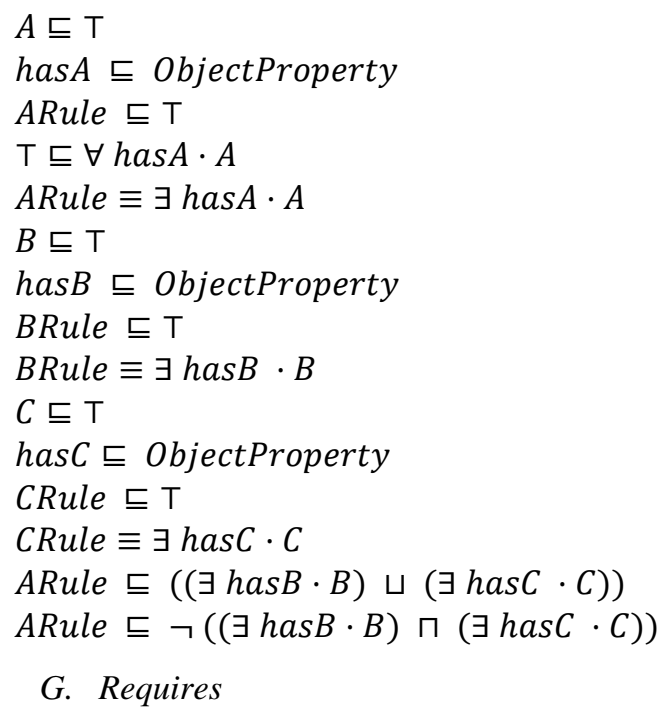

A feature may depend on some other features, hence its presence in a feature configuration requires the appearance of the others. For a given feature $A$ and a set of features $B_{1}, B_{2}, \ldots$, $B_{n}$ that $A$ requires, besides the $N S$ condition that binds ARule to $A$, we make sure that each of the $B i$ features appears in a configuration if $A$ is present.

$$
\text { ARule } \sqsubseteq \exists h a s B i \cdot B i \text { for } 1 \leq i \leq n
$$

\section{H. Excludes}

The presence of a feature may be inhibited by that of some other features. We say the appearance of a feature in a configuration excludes the appearance of some other features. For a given feature $A$ and a set of features $B 1, B 2, \ldots B n$ that $A$ excludes, we make sure, using the negation of someValuesFrom restriction on hasBi property, that Arule does not have any $B i$ feature.

$$
\text { ARule } ᄃ \neg(\exists h a s B i \cdot B i) \text { for } 1 \leq i \leq n
$$

We encoded the OWL-DL models of the Hall Booking System features into Protégé tool. Protégé gives an immediate feedback to the encoded logic by showing the corresponding feature graph ensuring the preliminary 
correctness of the syntax. Fig. 7 shows the feature graph created in Protégé.

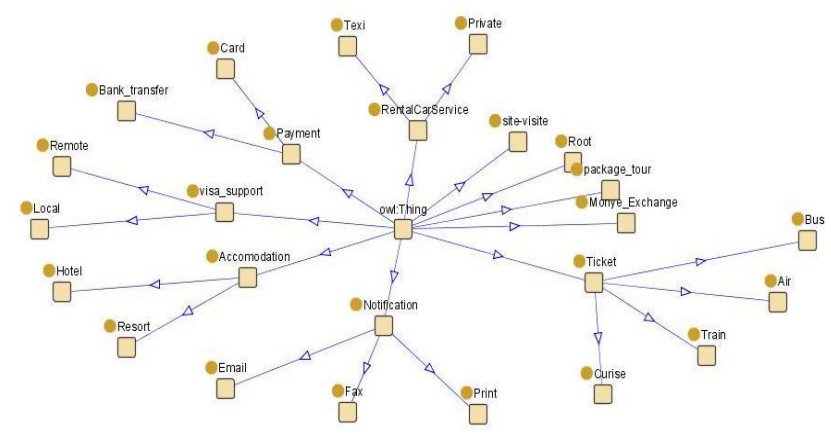

Fig. 7. Protégé screenshot.

\section{FEATURE VERIFICATION}

Consistency checking is a mechanism for checking whether the rules are semantically consistent. Consistency checking has to ensure that inconsistent requirements do not become part of the requirements specification of a given product. Our aim isto ensure that all the products derived from a feature model are consistent with requirements specifications. We can achieve this through the description and verification of semantic relationships between feature model and use scenarios.We input our ontology into Protégé and use RACER to check its consistency. For the initially encoded ontology, RACER checks for consistency and show that encoded definitions are consistent (Fig. 8).

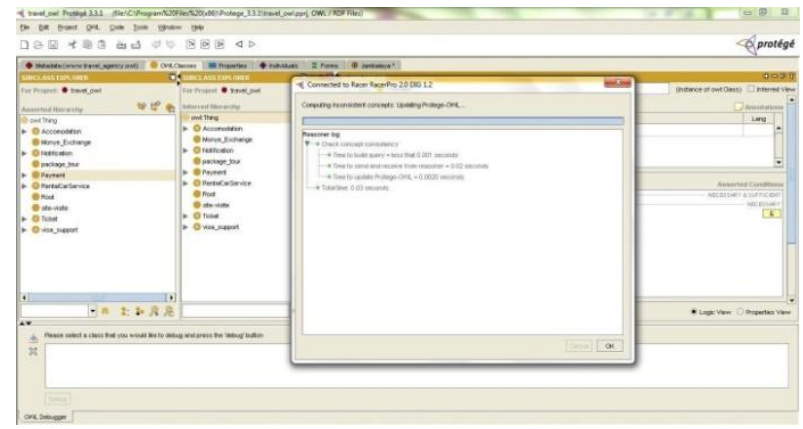

Fig. 8. Consistency checking in RACER.

In feature modeling, an instance of a concept is a configuration derived from the feature model. In order to detect inconsistency in a configuration OWL classes are used, and features and concept instances are then simulated. When an instance is checked, the reasoned tool not only can check inconsistency but also shows which class/classes are inconsistent. We use an existential restriction for each feature included in the configuration. For each feature $B$ available in a feature diagram but not in its configuration, we use a $\neg \exists$ has $B . B$ restriction to prevent the reasoning engine from inferring the existence of this feature in the configuration.

If an instance $C$ of a concept derived from a feature diagram with root concept $A$ and a set of features $B 1$, $B 2, \ldots, B i$ assuming that they appear in the configuration of $X$ and the features $B i+1, \ldots, B n$ do not, the feature configuration can be modeled as follows

$$
\begin{aligned}
& X \sqsubseteq \text { ARule } \\
& \left.\qquad X \equiv \Pi\left(\exists h a s B_{j} \cdot B j, \text { for } 1 \leq j \leq i\right)\right) \sqcap
\end{aligned}
$$

$$
\Pi\left(\neg \exists h a s B_{k} \cdot B_{k}, \text { for } i<k \leq n\right)
$$

\section{CONCLUSION}

Successful analysis and management of requirement plays a crucial role in the success of any software product. In this paper our emphasis is on the analysis of product line based web service composition. We explained that product line ideas can be successfully applied to web service to apply the reuse mechanism of software product line. We performed a detailed analysis of a travel agent web service in the light of software product line. Besides analyzing various feature requirement as well as service composition, we have also carried out a formal analysis of the feature diagram by applying semantic web technique

Our future plan is to extend the analysis model of the web service composition. In the goal model we only describe the success state of the goals. We also need to analyze the conditions for which a goal may fail. Analyzing the failed conditions we can improve the service composition. Our plan is to perform failure analysis and use any supporting tool to automate the analysis operations.

In OWL verification, we only performed consistency checking. We are also conducting experiment for instantiation checking for the web services. Our plan also includes representing the web service by using OWL-S which is a dialect of OWL to represent specifically web service compositions.

\section{REFERENCES}

[1] M. Burstein, C. Bussler, T. Finin, M. N. Huhns, M. Paolucci, A. P. Sheth, S. Williams, and M. Zaremba, "A semantic Web services architecture," IEEE Internet Computing, vol. 9, no. 5, pp. 72-81, 2005

[2] P. Oaks and A. terHofstede, "Guided interaction: A mechanism to enable ad hoc service interaction," Information Systems Frontiers, vol. 9, no. 1, pp. 29-51, 2007.

[3] B.-L. Tim et al., The semantic Web. Scientific American, vol. 284, no. 5, pp. 28-37, 2001

[4] W3C. Web Services Description Language (WSDL), Version 2.0, Part 1: Core Language, 2007.

[5] P. Clements and L. Northrop, Software Product Lines: Practices and Patterns, 3rd ed., Addison-Wesley Professional, 2001.

[6] I. Horrocks et al., "From shiq and rdf to owl: The making of a web ontology language," Journal of Web Semantics, vol. 1, 2003.

[7] N. F. Noy, M. Sintek et al., "Creating semantic web contents with protege-2000," Protégé-2000, IEEE Intelligent Systems, pp. 60-71, 2001.

[8] V. Haarslev and R. Möller, Racer user' guide and reference manual, version 1.7.6, 2002.

[9] K. C. Kang, S. G. Cohen, J. A. Hess, W. E. Novak, and A. S. Peterson, "Feature-oriented domain analysis (foda) feasibility study," Technical report, Carnegie-Mellon University Software Engineering Institute, November 1990.

[10] K. C. Kang, S. Kim, J. Lee, K. Kim, E. Shin, and M. Huh, "Form: A feature-oriented reuse method with domain-specific reference architectures," Ann. Softw. Eng., vol. 5, January 1998, pp. 143-168.

[11] K. Czarnecki and U. W. Eisenecker, Generative programming: methods, tools, and applications, New York, NY: ACM Press/Addison-Wesley Publishing Co., USA, 2000.

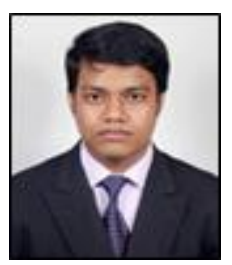

Nakul Chandra Das completed his B.Sc in compute science and engineering from East West University, Dhaka, Bangladesh in 2013. He is a member of Software Engineering and Formal Method Research Group, East West University. He is interested in web service composition, semantic web service, software engineering, and software product line. 


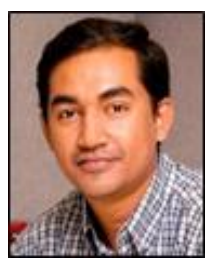

Shamim Ripon is an associate professor in the Department of Computer Science and Engineering, East West University, Dhaka, Bangladesh where he leads Software Engineering and Formal Method Research Group. Previously, he was a Research Associate in the Department of Computing Science, University of York,

UK and Research Fellow in the Department of Computing Science, University of Glasgow, UK. He also served as a lecturer in Khulna, University, Bangladesh. He is a member of IAENG, Senior member ofIACSIT.

Dr. Ripon holds a B.Sc. in computer science and engineering from Khulna University, MSc in Computer Science from National University of Singapore and $\mathrm{PhD}$ in Computer Science from University of Southampton, UK. His research interests focus on the requirement engineering, software product line, semantic web, natural language processing. His current research examines the formal representation and verification of knowledge based requirement specification.

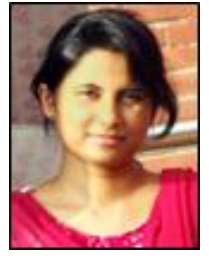

Orin Hossain completed her B.Sc in computer science and engineering from East West University in 2013. She is a member of Software Engineering and Formal Method Research Group, East West University. She is currently working towards formal verification of web services composition. She is also interested in semantic web based requirement analysis of product line requirements.

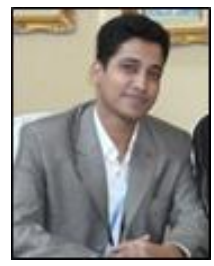

Mohammad Salah Uddin is a lecturer in the Department of Computer science and Engineering, Central Women's University, Dhaka, Bangladesh. He published several journals and international conference paper from his research work. He is a member of IACSIT and Software Engineering and Formal Method Research Group of East West University. He received his B.Sc in computer science and engineering from East West University, Dhaka, Bangladesh in 2012. He is interested in web service composition, semantic web service, knowledge representation, Mobile apps, Software Engineering, Software Product Line, Modeling and Verification. 